\title{
Adult Primary Intraventricular Hemorrhage: Clinical Characteristics and Outcomes
}

\author{
Aswin Pai ${ }^{1}$ Ajay Hegde ${ }^{1, \odot}$ Rajesh Nair ${ }^{1}$ Girish Menon ${ }^{1, \subseteq}$
}

Address for correspondence Girish Menon, MCh, DNB, FRCS, Department of Neurosurgery, Kasturba Medical College, Manipal Academy of Higher education, Manipal 576104, Karnataka, India (e-mail: girish.menon@manipal.edu).

\begin{abstract}
Keywords

- intraventricular hemorrhage

- intracerebral hemorrhage

- cerebral angiography

- hydrocephalus

- Graeb score

Background Adult primary intraventricular hemorrhage (PIVH) is a rare type of hemorrhagic stroke that is poorly understood. The study attempts to define the clinical profile, yield of diagnostic cerebral angiography, and prognosis of patients with PIVH. Patients and Methods Retrospective data analysis of all patients with PIVH admitted between February 2015 and February 2019 at a tertiary care center. Outcome was assessed using the modified Rankin scale (mRS) at 6 months.

Results and Discussion Our study group of 30 patients constituted 3.3\% (30/905) of our spontaneous intracerebral hemorrhage $(\mathrm{SICH})$ patients in the study period. The mean Glasgow Coma Score on admission was $11 \pm 3.33$ and the mean IVH Graeb score was 5.2 \pm 2.4 . All patients underwent angiography. Angiography detected moyamoya disease in four patients (13.3\%) and aneurysms in two patients (6.6\%) and these patients were managed surgically. Extraventricular drainage with intraventricular instillation of Streptokinase was performed in five patients. The rest of the patients was managed conservatively. At 6-month follow-up, 25 patients (83.33\%) achieved favorable outcome (mRS score of 0.1 or 2 ), whereas five (16.66\%) patients had a poor outcome (mRS score of 3 or more. Three patients succumbed to the illness. IVH Graeb score and presence of hydrocephalus have significant correlation with poor outcome. Conclusion PIVH is an uncommon entity but carries a better long-term prognosis than $\mathrm{SICH}$ angiography helps in diagnosing surgically remediable underlying vascular anomalies and is indicated in all cases of PIVH.
\end{abstract}

\section{Introduction}

Primary intraventricular hemorrhage (PIVH) accounts for $0.31 \%$ of all stroke cases and $3.1 \%$ of all spontaneous intracranial hemorrhage. ${ }^{1-6}$ PIVH refers to nontraumatic intracranial bleeding in the ventricular system and adjacent ependymal lining without a definite parenchymal component. ${ }^{7-10}$ $\mathrm{PIVH}$ is more commonly seen in preterm newborns than in adults. Secondary IVH refers to IVH that occurs with subarachnoid hemorrhage, or other parenchymal hemorrhage. The underlying etiopathogenesis for adult PIVH is variable and often remains idiopathic. The common associations include hypertension, arteriovenous malformations (AVMs), aneurysms, moyamoya disease (MMD), coagulopathy, and arteriovenous fistula., ${ }^{5,8,11-13}$ In this single-center retrospective study, we attempt to analyze the clinical profile pf patients with PIVH and evaluate the risk factors and prognosis of this relatively uncommon set of stroke patients. We also attempt to emphasize the need for angiography in all patients with PIVH.

\section{Patients and Methods}

We conducted a retrospective review of patients enrolled in our prospectively maintained intracerebral hemorrhage register from February 2015 to February 2019 after obtaining approval from our institutional ethics committee. Exclusion criteria were age $<18$ years patients with 
associated intraparenchymal, subarachnoid, or subdural hemorrhage, primary or secondary brain tumors with hemorrhage, hemorrhagic transformation of an ischemic stroke on initial computed tomography (CT) scan. PIVH was defined as blood present only in the ventricular system (lateral ventricle, third ventricle, fourth ventricle) with no associated parenchymal hemorrhage. All patients were admitted to a neurosurgical intensive care unit. In addition to the initial CT scans, all patients underwent vascular imaging in the form of computed tomography angiography (CTA) or magnetic resonance angiography (MRA), and/or digital subtraction angiography (DSA). The target for systolic blood pressure was $140 \mathrm{~mm} \mathrm{Hg}$. Osmotic diuretics were administered for 2 to 3 days and antiepileptic drugs were initiated to prevent secondary seizures. Patients with a Glasgow Coma Score (GCS) $<8$ were intubated and ventilated for airway protection. Fresh-frozen plasma and vitamin $\mathrm{K}$ was used to reverse abnormalities in coagulation if any. External ventricular drainage (EVD) was performed for all patients with a presenting GCS $<12$ with evidence of hydrocephalus. Intraventricular instillation of Inj. Streptokinase 30,000 IU daily was done for all patients with EVD. Pneumatic compression stockings were used to prevent deep vein thrombosis. Gender, age, GCS on admission, length of stay, presenting symptoms, pre-existing medical conditions, imaging findings, and outcomes were analyzed from the subject's medical records. Volume of IVH was calculated using the modified Graeb score system. ${ }^{14}$ Hydrocephalus was defined as an Evans' ratio of $>0.30 .^{1,2,10}$ Neurological outcome at 3 and 6 months was assessed using the modified Rankin scale (mRS) and a favorable outcome group was defined as mRS scores of 3 or less and an unfavorable outcome group as mRS scores of 4 or above. Descriptive statistics were performed using SPSS version 19 (Chicago, Illinois, United States).

\section{Results}

From February 2015 to February 2019, a total of 905 patients were admitted to our department with SICH. Of these 455 patients (50.28\%) had evidence of IVH on initial noncontrast CT scan. PIVH (with no evidence of parenchymal or subarachnoid hemorrhage) was seen in 30 patients (3.3\%). This cohort formed our study group. The remaining 425 (47\%) patients had secondary IVH (associated parenchymal or subarachnoid hemorrhage). The study group included 20 men $(66.66 \%)$ and 10 women $(33.33 \%)$ with a mean age of $49.9 \pm 12.3$. The common presenting symptoms were sudden onset headache ( $n=17,56.66 \%)$, vomiting ( $n=5$, $16.66 \%)$, and altered sensorium ( $n=12,40 \%)$. The mean GCS score on admission was $11 \pm 3.33$ and the mean IVH Graeb score was $5.2 \pm 2.4$. Past history of hypertension was identified in 17 patients (56.66\%), hyperlipidemia in 7 (23.33\%), diabetes in 10 (33.33\%), smoking in 6 (20\%), and chronic alcoholism in $3(10 \%)$ patients, respectively. Coagulopathy caused by antiplatelet and anticoagulants was observed in five and two patients, respectively. Patient demographics and clinicoradiological details are shown in - Table $\mathbf{1}$.
Of our 30 patients, 20 underwent DSA, 10 underwent CTA and 5 underwent both CTA and MRA. The etiological causes identified by angiography included MMD in four patients (13.3\%) (-Fig. 1) and aneurysm in two patients (6.6\%) (-Fig. 2). The aneurysms were located in the distal anterior cerebral and posterior inferior cerebellar location. In our series, five patients with hydrocephalus and a GCS $<8$ were managed with an EVD with daily administration of 30,000 IU of Inj. Streptokinase for 5 days. Only one of these five patients had a good outcome. Two patients with proven aneurysms underwent clipping. Patients with MMD underwent combined revascularization procedures including both direct superficial temporal artery to middle cerebral artery (STMC) anastomosis along with encephaloduroarteriomyosynangiosis. All the other remaining patients were managed conservatively. At 30-day follow-up, 23 patients (76.66\%) achieved favorable outcome (mRS score of 0.1 or 2 ), whereas seven patients $(23.33 \%$ ) had a poor outcome (mRS score of 3 or more). At 6-month follow-up, 25 patients (83.33\%) achieved favorable outcome (mRS score of 0.1 or 2 ), whereas $5(16.66 \%$ ) patients had a poor outcome (mRS score of 3 or more). One patient with hemorrhagic MMD had a stormy postoperative course and succumbed following prolonged ventilation and sepsis. Two other patients with poor Graeb score and acute hydrocephalus succumbed in spite of aggressive antiedema measures.

On univariate analysis, Graeb score and hydrocephalus were found to have significant correlation with poor outcome. On multivariate analysis, only hydrocephalus had significant correlation with poor outcome. All the other

Table 1 Patient demographics and clinicoradiological details

\begin{tabular}{|c|c|}
\hline \multicolumn{2}{|c|}{ Total number of patients: 30} \\
\hline Age, years (mean \pm SD) & $49.9 \pm 12.3$ \\
\hline \multicolumn{2}{|l|}{ Gender } \\
\hline Male & $66.66 \%(n=20)$ \\
\hline Female & $33.33 \%(10)$ \\
\hline \multicolumn{2}{|l|}{ Initial symptoms } \\
\hline Headache & $56.66 \%(17)$ \\
\hline Vomiting & $16.66 \%(5)$ \\
\hline Altered sensorium & $40 \%(12)$ \\
\hline GCS score(mean) & $11+\mid-3.33$ \\
\hline \multicolumn{2}{|l|}{ Comorbidities } \\
\hline Hypertension & $56.66 \%(17)$ \\
\hline Antiplatelet & $16.66 \%(5)$ \\
\hline Anticoagulants & $6.66 \%(2)$ \\
\hline \multicolumn{2}{|l|}{ IVH score } \\
\hline Graeb score & 5.22 .4 \\
\hline \multicolumn{2}{|l|}{ Etiology } \\
\hline Moyamoya & $13.33 \%(4)$ \\
\hline Aneurysm & $6.66 \%(2)$ \\
\hline Idiopathic & $23.39 \%(7)$ \\
\hline
\end{tabular}

Abbreviations: GCS, Glasgow Coma Score; IVH, intraventricular hemorrhage; SD, standard deviation. 

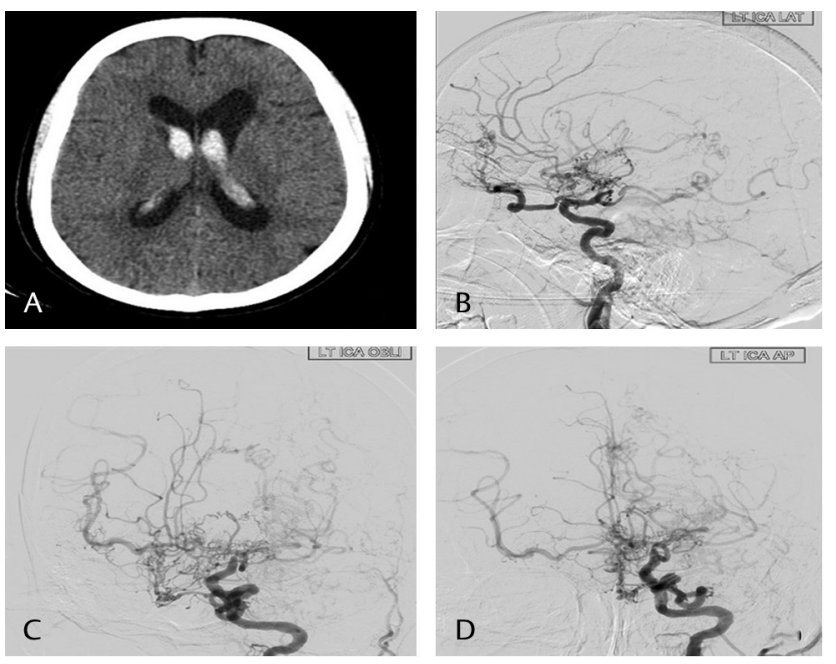

Fig. 1 Computed tomography scan plain axial image (A) showing intraventricular hemorrhage in the lateral ventricle. Digital subtraction angiography images of left internal carotid artery-lateral (B), oblique (C), and anteroposterior (D) showing presence of moyamoya vessels and complete obliteration of the supraclinoid internal carotid artery.

Table 2 Outcome prognosticators

\begin{tabular}{|c|c|c|c|c|}
\hline \multirow[t]{2}{*}{ Total number of patients: 30} & \multirow{2}{*}{$\begin{array}{l}\text { Favorable outcome } \\
\mathrm{mRS}<3\end{array}$} & \multirow{2}{*}{$\begin{array}{l}\text { Poor outcome } \\
\mathrm{mRS}>3\end{array}$} & \multicolumn{2}{|c|}{ Univariate analysis } \\
\hline & & & $p$-Value & OR \\
\hline \multicolumn{5}{|l|}{ Age } \\
\hline Age, years $(\text { mean } \pm S D)^{a}$ & $48.48 \pm 11.85$ & $60 \pm 14.61$ & 0.08 & 0.92 \\
\hline \multicolumn{5}{|l|}{ Sex } \\
\hline Male & 17 & 3 & 0.72 & 0.7 \\
\hline Female & 8 & 2 & & \\
\hline \multicolumn{5}{|l|}{$\mathrm{GCS}^{\mathrm{b}}$} \\
\hline GCS $13-15$ & 14 & 1 & & \\
\hline GCS 9-12 & 9 & 1 & 0.43 & 0.37 \\
\hline GCS $<8$ & 2 & 3 & & \\
\hline \multicolumn{5}{|l|}{ Comorbidities } \\
\hline Hypertension & 14 & 3 & 0.86 & 0.84 \\
\hline Antiplatelet & 2 & 3 & 0.82 & 0.76 \\
\hline Anticoagulants & 1 & 1 & 0.19 & 0.16 \\
\hline \multicolumn{5}{|l|}{ Radiology } \\
\hline Graeb $^{\mathrm{b}}$ & $4.15 \pm 2.06$ & $7.4 \pm 1.4$ & 0.05 & 0.32 \\
\hline Acute hydrocephalus & 1 & 4 & 0.01 & 0.05 \\
\hline \multicolumn{5}{|l|}{ Etiology } \\
\hline Moyamoya & 3 & 1 & 0.63 & 0.54 \\
\hline Aneurysm & 1 & 1 & 0.19 & 0.16 \\
\hline Idiopathic & 4 & 3 & 0.06 & 0.12 \\
\hline
\end{tabular}

Abbreviations: GCS, Glasgow Coma Score; mRS, modified Rankin scale; OR, odds ratio; SD, standard deviation. andependent sample T-test.

${ }^{b}$ Mann-Whitney $U$ test, Rest are chi-squared test.
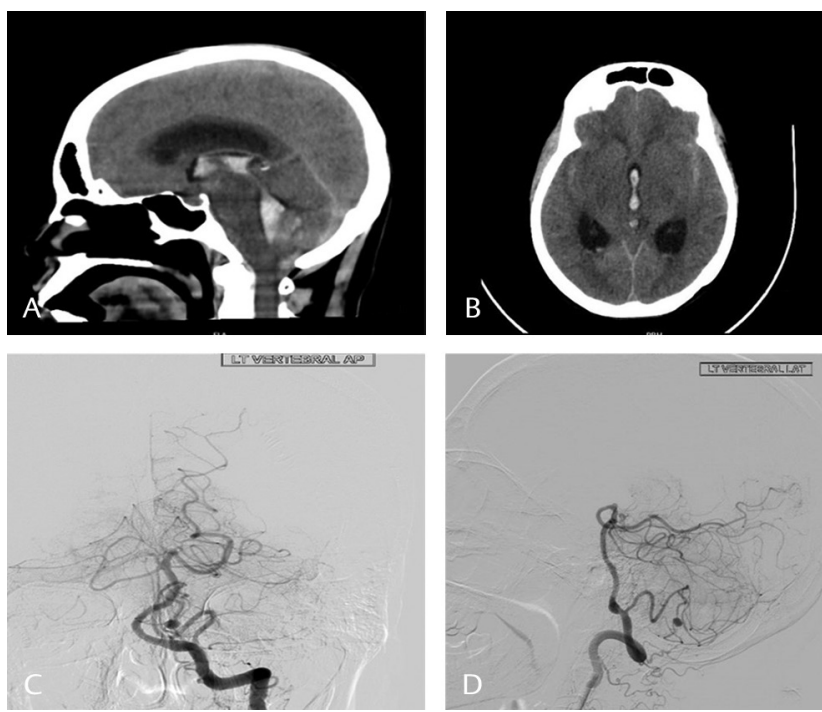

Fig. 2 Computed tomography scan plain sagittal (A) and axial (B) images showing presence of blood in the third ventricle and the fourth ventricle. Digital subtraction images vertebral artery anteroposterior (C) and lateral (D) showing the presence of a posterior inferior cerebellar artery aneurysm. 
variables did not have any statistically significant influence on outcome (-Table 2 ).

\section{Discussion}

IVH has been associated with an ominous outcome in patients with spontaneous intracerebral hemorrhage (SICH). However, PIVH, unlike secondary IVH, carries a better prognosis. Due to the small number of reported cases, there is a limited understanding of its enteropathogenesis and outcome. ${ }^{1,9,15-17}$

The incidence of PIVH varies from 1 to $8.8 \%$ of all cases of spontaneous intracranial hemorrhage., ${ }^{5,16,18-21}$ The variation may be explained by the fact that conventional CT scan with 5 to $10 \mathrm{~mm}$ cuts in thickness may leave a small volume of intraparenchymal hemorrhage undetected. This often results in overestimation unless thin slice CT scan images are studied. ${ }^{15,22}$ In our study, PIVH accounted for $3.3 \%$ of all SICH patients. ${ }^{23}$ This corresponds to the published observation that PIVH accounts for $0.31 \%$ of stroke cases and $3.1 \%$ of all spontaneous intracranial hemorrhage in adults. ${ }^{24,25}$

It is generally believed that the incidence of PIVH increases with age. ${ }^{3,26}$ In Arboix's study, the mean age of patients with PIVH was 78.9 years and $41.7 \%$ of patients were aged 85 years or above. ${ }^{9}$ The mean age of 50 years observed in our series is much lower than what has been reported in other series. This relatively younger age at presentation was observed in our primary SICH series too and has been reported in other $\mathrm{SICH}$ series from the Indian subcontinent ${ }^{23}$. A similar trend has been reported in the series published by Mart 1-Fabregas et al and Hameed et al where the mean age was considerably lower-60 and 56 years, respectively. ${ }^{7,17}$ Patients with underlying vascular pathologies causing PIVH tend to be younger than those without any angiographic abnormality.,12,13 The mean age of patients with proven vascular anomalies such as MMD and aneurysms was 41 years in our series which conforms to the reported observation. Gender predilection in PIVH is variable and both male and female predominance have been equally reported. ${ }^{1,2,24}$ We observed a distinct male preponderance (2:1 ratio) that could be probably related to the prevailing socioeconomic factors in India and not a true reflection of male preponderance.

Sudden onset headache followed by loss of consciousness is the commonest presenting feature of PIVH. Headache has been postulated to be secondary to chemical stimulation and mass effect of the hematoma with ventricular dilatation. ${ }^{23}$ The mean GCS of the patients without hydrocephalus in our series was 13 . This probably could be attributed to the fact that the ventricle tends to accommodate and expand with blood.

The exact etiopathogenesis of IVH remains unclear. The source of bleeding in PIVH can be identified in only around $56 \%$ of patients and the etiology remains unknown in most of the studies. Such patients with PIVH without any detectable underlying structural cerebrovascular abnormalities are referred to as idiopathic PIVH. Although a history of hypertension is a common finding, its association with PIVH has not been consistently observed in all studies and varies from
38.4 to $80 \%{ }^{11}$ Chronic hypertension is believed to induce hemorrhage in choroidal arteries that probably results in PIVH. ${ }^{7}$ In Guo et al's series of 98 patients, the incidence of idiopathic PIVH was $66.2 \%$ and hypertension was considered to be the primary risk factor, occurring in $62.24 \%$ of patients. ${ }^{1}$ In Zhang's series, idiopathic PIVH constituted $47.7 \%$ of all PIVH patients, and hypertension was considered to be the primary predisposing factor. ${ }^{5}$ Idiopathic IVH probably related to systemic hypertension constituted a little over half of the patients (56.6\%; 17 patients) in our series. Vascular anomalies such as aneurysms, AVMs, account for around $30 \%$ of PIVH. One meta-analysis of a series of 14 case series including 339 PIVH patients reported the presence of AVM, aneurysms, and MMD as 31, 22, and 3\%, respectively. Other rare vascular etiologies for PIVH include lenticulostriate aneurysms, basilar and carotid arterial dissection, and dural arteriovenous fistula. ${ }^{12,13,15,19} \mathrm{PIVH}$ due to anticoagulants or antiplatelet is unusual. ${ }^{27}$ In our series, five patients gave history of antiplatelet usage and two were on anticoagulants. Bleeding and coagulation parameters in these patients were however normal on admission and the exact causative nature of the association is difficult to establish.

The need for angiography in all cases of PIVH is debatable. ${ }^{10,12,19,25}$ In our series, all the patients underwent angiography. Angiography (either CTA or DSA) was able to pick up two cases of aneurysm and four cases of MMD in our small series of 30 patients. Our incidence of 13.3\% MMD is a reflection of the fact that MMD is being increasingly detected in India of late. The diagnostic yield in other series has varied from 30 to $60 \%{ }^{10,19}$ The variation may be explained by the fact that all patients in these series did not undergo angiography. We observed that in our series patients who had a positive angiogram tend to be younger (mean age of 41 years) and had less incidence of hypertension (16.66\%) when compared with those who were angio negative (mean age 52.75, hypertensive [66.66\%]). We strongly believe that although the chances of a positive finding are minimal, angiogram helps in identifying a treatable underlying ethology and should be performed in all cases of PIVH. CTA or MRA may help to screen patients but DSA remains the gold standard to pick up subtle underlying etiologies such as small choroidal aneurysms. Patients without hypertension, coagulopathies, or any vascular anomalies constitute a special entity of PIVH, and occur in 6.7 to $38.5 \%$ of PIVH patients. The postulated mechanism for bleeds in such patients could be rupture of small vascular malformations on the ventricular wall or lenticulostriate microaneurysms, which could vanish after bleeding. In our study, etiology was unknown in $23.39 \%$. However, presence of vascular lesion cannot be always ruled out by angiogram in acute stage as small aneurysms or AVMs may be compressed by hematoma and hence may not be detected.

Treatment paradigms vary depending on the volume of bleed, presence of hydrocephalus, and GCS of the patient. Recommended supportive medical treatments include antiedema measures and antihypertensive agents. Use of EVD is controversial and is generally guided by the presence of hydrocephalus and clinical condition of the patient. ${ }^{28}$ In adults, intraventricular thrombolytic therapy 
with recombinant tissue plasminogen activator and other fibrinolytics is known to facilitate resolution of IVH that reduces intracranial pressure. It also reduces the duration of cerebrospinal fluid diversion, and ameliorate direct neural injury. ${ }^{29-31}$ Many studies and trials have been conducted to prove the efficacy of intraventricular thrombolytic therapy. However, most of these studies have concentrated on secondary IVH. Coplin et al studied 40 patients with IVH of whom 22 patients received adjunctive intraventricular urokinase together with ventriculostomy. ${ }^{29}$ The group treated with urokinase had a lower mortality with an increasing trend toward favorable outcome. In the 10 patients treated by Findlay et al, they concluded that recombinant tissue plasminogen activator improved overall outcome by facilitating rapid lysis of the IVH and drainage with normalization of ventricular size and intracranial pressure. ${ }^{30}$ In another review of seven independent studies using thrombolytic agents, good outcomes were observed in 50 of the 74 patients $^{32}$.The CLEAR-IVH trial (parts $A$ and $B$ ) showed that use of low-dose tissue plasminogen activator may increase lysis rates. ${ }^{33}$ However, hemorrhagic complications have remained a concern and intraventricular thrombolysis is yet to be accepted as standard of care both in secondary and PIVH. In addition, EVD also carries a risk of infection and currently there is insufficient evidence to support its use in all cases. We observed that in the five patients who underwent thrombolysis in our series, resolution of IVH was better but that did not translate into good clinical outcome.

Outcome following PIVH is reported to be better than those with secondary IVH. . $7,8,16,26$ The lack of parenchymal damage in PIVH is believed to be responsible for this difference. Mortality in the first week is high in patients presenting with poor GCS. ${ }^{3,4,34}$ Thereafter, patients gradually recover and the outcome becomes progressively better. In Lee's study while $16 \%$ of the patients died within a week of ictus, $64 \%$ of the remaining patients improved and had favorable outcomes at 3 months. ${ }^{16}$ Guo et al also reported an overall favorable outcome in their cohort, with a mean mRS score of $1.40 \pm 1.38$ and mortality of $4 \% .^{1}$ Our observations are similar with a $10 \%$ mortality and a good outcome ( $\mathrm{mRS}<3$ ) in nearly 83.33\% at the end of 6 months.

In the absence of large series, outcome prognosticators following PIVH are not well defined. GCS on admission $($ GCS $<8)$ is the most consistent prognostic factor in almost all series. ${ }^{10,17,21,35}$ In addition, age, volume of intraventricular hematoma (Graeb score), the associated subarachnoid, hydrocephalus, and brain atrophy are all important prognostic factors. Of these, age and the volume of IVH are independent preceptors of in-hospital mortality. ${ }^{19}$ While IVH may directly not be responsible for mortality, it is the hemorrhagic dilation of the fourth ventricle, followed by a sudden increase in intraventricular pressure, which results in irreversible ischemic injury to the brain stem, resulting in death. ${ }^{16}$ Coagulopathy is believed to be another negative prognosticator and correction of the hematological parameters is reported to improve outcome. Intensive antihypertensive treatment during the acute phase has been noted to improve clinical outcomes in some studies, an observation which we could not establish in our study. Similarly, neither the presence of causative vascular abnormalities nor the treatment of the underlying lesions is known to affect the short-term clinical outcome of PIVH. In our series, all the six patients with underlying vascular anomalies underwent surgery and the outcome was variable. One of the four MMD patients and one aneurysm patient had a poor outcome and the rest of the patients had a good outcome. In our study, low GCS and higher age were found to be associated with poor outcome but without statistical significance. Graeb score and hydrocephalus were found to have significant correlation with poor outcome on univariate analysis. On multivariate analysis, only hydrocephalus had significant correlation with poor outcome.

\section{Conclusion}

PIVH is uncommon but carries a more favorable prognosis than secondary IVH. Systemic hypertension is a common association but its etiological role in PIVH is unclear. Angiography helps in diagnosing surgically treatable underlying vascular anomalies and should be performed in all cases of PIVH. Presence of hydrocephalus and a high Graeb score indicate poor prognosis.

\section{Funding}

None.

\section{Conflict of Interest}

None declared.

\section{References}

1 Guo R, Ma L, Shrestha BK, Yu Z, Li H, You C. A retrospective clinical study of 98 adult idiopathic primary intraventricular hemorrhage cases. Medicine (Baltimore) 2016;95(42):e5089

2 El-Saadany WF, Hassan T. Adult intraventricular hemorrhage: presentations, management, and analysis of outcome. Neurosurg Q 2012;22(1):30-37

3 Passero S, Ulivelli M, Reale F. Primary intraventricular haemorrhage in adults. Acta Neurol Scand 2002;105(2):115-119

4 Angelopoulos M, Gupta SR, Azat Kia B. Primary intraventricular hemorrhage in adults: clinical features, risk factors, and outcome. Surg Neurol 1995;44(5):433-436, discussion 437

5 Zhang S, Jia B, Li H, You C, Hanley DF, Jiang Y. Primary intraventricular hemorrhage in adults: etiological causes and prognostic factors in Chinese population. J Neurol 2017;264(2):382-390

6 Gates PC, Barnett HJ, Vinters HV, Simonsen RL, Siu K. Primary intraventricular hemorrhage in adults. Stroke 1986;17(5):872-877

7 Martí-Fàbregas J, Piles S, Guardia E, Martí-Vilalta J-L. Spontaneous primary intraventricular hemorrhage: clinical data, etiology and outcome. J Neurol 1999;246(4):287-291

8 Giray S, Sen O, Sarica FB, et al. Spontaneous primary intraventricular hemorrhage in adults: clinical data, etiology and outcome. Turk Neurosurg 2009;19(4):338-344

9 Arboix A, García-Eroles L, Vicens A, Oliveres M, Massons J. Spontaneous primary intraventricular hemorrhage: clinical features and early outcome. ISRN Neurol 2012;2012(Aug):498303 
10 Srivastava T, Sannegowda RB, Satija V, Jain RS, Tejwani S, Mathur T. Primary intraventricular hemorrhage: clinical features, risk factors, etiology, and yield of diagnostic cerebral angiography. Neurol India 2014;62(2):144-148

11 Brott $\mathrm{T}$, Thalinger $\mathrm{K}$, Hertzberg V. Hypertension as a risk factor for spontaneous intracerebral hemorrhage. Stroke 1986;17(6):1078-1083

12 Mohr G, Ferguson G, Khan M, et al. Intraventricular hemorrhage from ruptured aneurysm. Retrospective analysis of 91 cases. J Neurosurg 1983;58(4):482-487

13 Padmanabhan R, Stacey R, Wimalaratna S, Kuker W. Dural arteriovenous fistula causing primary intraventricular haemorrhage. Br J Radiol 2008;81(962):e44-e47

14 Morgan TC, Dawson J, Spengler D, et al; CLEAR and VISTA Investigators. The Modified Graeb Score: an enhanced tool for intraventricular hemorrhage measurement and prediction of functional outcome. Stroke 2013;44(3):635-641

15 Graeb DA, Robertson WD, Lapointe JS, Nugent RA, Harrison PB. Computed tomographic diagnosis of intraventricular hemorrhage. Etiology and prognosis. Radiology 1982;143(1):91-96

16 Lee S-H, Park K-J, Park D-H, Kang S-H, Park J-Y, Chung YG. Factors associated with clinical outcomes in patients with primary intraventricular hemorrhage. Med Sci Monit 2017;23:1401-1412

17 Hameed B, Khealani BA, Mozzafar T, Wasay M. Prognostic indicators in patients with primary intraventricular haemorrhage. J Pak Med Assoc 2005;55(8):315-317

18 Little JR, Blomquist GA Jr, Ethier R. Intraventricular hemorrhage in adults. Surg Neurol 1977;8(3):143-149

19 Flint AC, Roebken A, Singh V. Primary intraventricular hemorrhage: yield of diagnostic angiography and clinical outcome. Neurocrit Care 2008;8(3):330-336

20 Darby DG, Donnan GA, Saling MA, Walsh KW, Bladin PF. Primary intraventricular hemorrhage: clinical and neuropsychological findings in a prospective stroke series. Neurology 1988;38(1):68-75

21 Jayakumar PN, Taly AB, Bhavani UR, Arya BY, Nagaraja D. Prognosis in solitary intraventricular haemorrhage. Clinical and computed tomographic observations. Acta Neurol Scand 1989;80(1):1-5

22 McCallum JE, LoDolce D, Boehnke M. CT scan in intraventricular hemorrhage: correlation of clinical findings with computerized tomographic scans of the brain. Neurosurgery 1978;3(1):22-25
23 Hegde A, Menon G. Modifying the intracerebral hemorrhage score to suit the needs of the developing world. Ann Indian Acad Neurol 2018;21(4):270-274

24 Weinstein R, Ess K, Sirdar B, Song S, Cutting S. Primary intraventricular hemorrhage: clinical characteristics and outcomes. J Stroke Cerebrovasc Dis 2017;26(5):995-999

25 Zhu XL, Chan MS, Poon WS. Spontaneous intracranial hemorrhage: which patients need diagnostic cerebral angiography? A prospective study of 206 cases and review of the literature. Stroke 1997;28(7):1406-1409

26 Radic JAE, Vincer M, McNeely PD. Outcomes of intraventricular hemorrhage and posthemorrhagic hydrocephalus in a population-based cohort of very preterm infants born to residents of Nova Scotia from 1993 to 2010. J Neurosurg Pediatr 2015; 15(6):580-588

27 Biffi A, Battey TWK, Ayres AM, et al. Warfarin-related intraventricular hemorrhage: imaging and outcome. Neurology 2011;77(20):1840-1846

28 Kiymaz N, Demir O, Çirak B. Is external ventricular drainage useful in primary intraventricular hemorrhages? Adv Ther 2005;22(5):447-452

29 Coplin WM, Vinas FC, Agris JM, et al. A cohort study of the safety and feasibility of intraventricular urokinase for nonaneurysmal spontaneous intraventricular hemorrhage. Stroke 1998;29(8):1573-1579

30 Findlay JM, Grace MG, Weir BK. Treatment of intraventricular hemorrhage with tissue plasminogen activator. Neurosurgery 1993;32(6):941-947, discussion 947

31 Todo T, Usui M, Takakura K. Treatment of severe intraventricular hemorrhage by intraventricular infusion of urokinase. J Neurosurg 1991;74(1):81-86

32 Lapointe M, Haines S. Fibrinolytic therapy for intraventricular hemorrhage in adults. Cochrane Database Syst Rev 2002;(3):CD003692

33 Morgan T, Awad I, Keyl P, Lane K, Hanley D. Preliminary report of the clot lysis evaluating accelerated resolution of intraventricular hemorrhage (CLEAR-IVH) clinical trial. Acta Neurochir Suppl (Wien) 2008;105:217-220

34 Verma A, Maheshwari MC, Bhargava S. Spontaneous intraventricular haemorrhage. J Neurol 1987;234(4):233-236

35 Park KJ, Kim JH, Park YK, Chung HS, Lee HG, Suh JK. Prognostic Factors of Primary Intraventricular Hemorrhage. J Korean Neurosurg Soc 2004;35(3):278-283 